\title{
Perfil epidemiológico de las intoxicaciones por antidepresivos recibidas en el Centro Información y Asesoramiento Toxicológico uruguayo en el período 2010-2012
}

Carolina Amigo*, María Noel Tortorella†, Viviana Domínguez*, Noelia Speranza*, Amalia Laborde ${ }^{\dagger}$, Gustavo Tamosiuna*

\section{Resumen}

Introducción: la depresión es una de las principales causas mundiales de discapacidad. Datos nacionales mostraron que 10,6\% de la población consumió en alguna oportunidad algún antidepresivo. La importancia de la exposición de este grupo terapéutico en la sociedad se puede ver reflejada, entre otros aspectos, en la frecuencia en que se encuentran implicados en intoxicaciones.

Objetivo: el objetivo de este estudio fue describir el perfil epidemiológico de las intoxicaciones por antidepresivos recibidas por el Centro de Información y Asesoramiento Toxicológico (CIAT) entre los años 2010 y 2012.

Metodología: estudio observacional retrospectivo en el cual se analizaron las consultas de intoxicaciones que involucraron un antidepresivo, registradas en el CIAT entre 2010-2012.

Resultados: se recibieron 32.565 consultas toxicológicas en el período analizado. Los antidepresivos fueron implicados como agente tóxico causal en 2.523 (7,7\%). La mayoría de las intoxicaciones ocurrieron en pacientes de sexo femenino $(75,4 \%)$, adultos $(79,3 \%)$ y fueron de causa intencional (90\%). El antidepresivo más frecuentemente implicado fue sertralina (38\%). La totalidad de las intoxicaciones severas $(54 ; 2,1 \%)$ implicaron más de un fármaco. Se registró un solo caso fatal.

Conclusiones: el perfil de intoxicaciones por antidepresivos en Uruguay entre 2010 y 2012 es similar al reportado en series internacionales y apoya el ya conocido riesgo de los antidepresivos de segunda generación. Si bien en número escaso, se destaca la exposición a estos medicamentos en menores de 18 años, sobre todo menores de 5 años, lo que requiere mayor caracterización.

Palabras clave: Antidepresivos

Envenenamiento

Factores de riesgo

Key words: Antidepressive Agents

Poisoning

Risk factors

\footnotetext{
* Departamento de Farmacología y Terapéutica. Hospital de Clínicas, Facultad de Medicina, Universidad de la República, Uruguay. † Centro de Información y Asesoramiento Toxicológico (CIAT), Departamento de Toxicología. Hospital de Clínicas, Facultad de Medicina, Universidad de la República, Uruguay.

Recibido: $2 / 5 / 18$

Aprobado: $15 / 8 / 18$
} 


\section{Introducción}

La depresión mayor es una de las principales causas de discapacidad a nivel mundial. Se estima que 350 millones de personas en el mundo la padecen, según datos registrados por la Organización Mundial de la Salud (OMS) en el año 2012 ${ }^{(1)}$. En Estados Unidos afecta a más de 14,8 millones de personas y es la principal causa de discapacidad en adolescentes y adultos jóvenes. La media de edad en la que se registra esta patología son los 32 años y es más prevalente en mujeres ${ }^{(2)}$. En Uruguay se encontró un aumento de su prevalencia de $11 \%$ en 1998 a $15 \%$ en $2008^{(3)}$.

La base biológica de la depresión se ha explicado tradicionalmente por un déficit cuantitativo en las monoaminas biológicas (serotonina, noradrenalina, dopamina), por este motivo los medicamentos antidepresivos se han clasificado según su mecanismo para mejorar la transmisión monoaminérgica ${ }^{(4)}$. Los primeros fármacos relativamente específicos como tratamiento antidepresivo fueron los inhibidores de la monoamino oxidasa (IMAO), los cuales inicialmente se sintetizaron como medicamentos antituberculosos, pero que finalmente demostraron mejorar sustancialmente el estado de ánimo de estos pacientes. Posteriormente se introdujeron los antidepresivos tricíclicos (ADT), que constituyeron el pilar principal del tratamiento para la depresión hasta la aparición en el mercado en los años 90 de los inhibidores selectivos de la recaptación de serotonina (ISRS). La principal diferencia entre ambos grupos es su perfil de riesgo ${ }^{(5)}$. Actualmente se encuentran disponibles en el mercado también los inhibidores selectivos de la recaptación de serotonina y noradrenalina, denominados antidepresivos de acción "dual"(4).

Las indicaciones principales de los antidepresivos son el tratamiento de los trastornos depresivos y los trastornos de ansiedad, sin embargo algunos también están autorizados para el tratamiento del dolor crónico neuropático o la deshabituación del hábito tabáquico ${ }^{(5-11)}$.

En relación directa al creciente aumento de la prevalencia de esta patología, y con la síntesis de nuevos antidepresivos que se postulan como más seguros, es que se ha registrado en los últimos tiempos un aumento en el consumo de estos medicamentos a nivel mundial. Por ejemplo, en España, la Agencia Española de Medicamentos y Productos Sanitarios (AEMPS) informó un aumento de 200\% en el consumo desde el año 2000 al $2013^{(12)}$; en Inglaterra se ha constatado un aumento de $165 \%$ en la prescripción de antidepresivos entre 1998 y $2012^{(13)}$. A nivel regional, en Chile se registró un aumento del consumo de antidepresivos de $470 \%$ desde 1992 a $2004^{(14)}$.

Datos preliminares provenientes de la Sexta Encuesta Nacional en Hogares sobre Consumo de Drogas de
Uruguay, publicados en mayo de 2015, mostraron que $10,6 \%$ de la población encuestada consumió en alguna oportunidad algún antidepresivo en su vida ${ }^{(15)}$, en comparación con $6,6 \%$ registrado en la encuesta previa publicada en el año $2012^{(16)}$.

La importancia de la exposición de este grupo terapéutico en la sociedad se puede ver reflejada, entre otros aspectos, en la frecuencia en que los antidepresivos se encuentran implicados en intoxicaciones. En las series internacionales y regionales consultadas la incidencia de intoxicaciones por este grupo terapéutico se encuentra entre 3\% y $17 \%$, según el país y el área asistencial donde se realiza el análisis ${ }^{(17-20)}$.

La intoxicación aguda por antidepresivos puede causar toxicidad en el sistema nervioso central (SNC) y a nivel cardíaco, siendo esta última responsable de la mortalidad como resultado de la sobredosis, sobre todo con los antidepresivos más antiguos ${ }^{(21)}$. La clínica de intoxicación aguda por ISRS es una extensión directa de su actividad farmacológica, produciendo un exceso de estimulación serotoninérgica prominente y no selectiva. Los antidepresivos atípicos o duales son antidepresivos más nuevos, la mayoría derivados de ISRS con un efecto farmacológico adicional seleccionado para disminuir efectos adversos de los antidepresivos tradicionales, pero en sobredosis algunos de ellos también causan convulsiones y toxicidad cardíaca ${ }^{(22)}$.

En un trabajo previo publicado por los autores en 2015, en el que se describen las intoxicaciones por benzodiazepinas, se discute que el aumento a la exposición de estos fármacos no solo es significativa del punto de vista cuantitativo (por el aumento de su consumo), sino también por sus determinantes y los potenciales riesgos que éste implica (por uso irracional y falta de percepción de riesgo, por ejemplo) ${ }^{(23)}$.

Basados en el aumento del consumo de antidepresivos a nivel internacional antes descrito y la falta de información en nuestro medio sobre intoxicaciones, se plantea la realización de este trabajo con el objetivo de describir el perfil epidemiológico de las intoxicaciones por antidepresivos recibidas en el Centro de Información y Asesoramiento Toxicológico (CIAT), centro de referencia nacional en intoxicaciones, entre los años 2010 y 2012.

\section{Metodología}

Se realizó un estudio observacional retrospectivo en el cual se identificaron, cuantificaron y analizaron todas las consultas de intoxicaciones que involucraron un agente antidepresivo, registradas en el CIAT entre los años 2010 y 2012. Los registros se obtuvieron de la base de datos del sistema INTOX (Data Management System) que permite ingresar la historia clínica genera- 
da en las consultas al médico de guardia del CIAT. La base de datos INTOX fue manejada por uno de los autores integrante del CIAT.

A los fines del presente trabajo se considera "consulta toxicológica" a toda consulta recibida por los médicos del CIAT en forma telefónica o por interconsulta dentro del Hospital de Clínicas en Montevideo (hospital donde se ubica geográficamente el CIAT), "agente tóxico" a cualquier sustancia que el médico del CIAT registra como asociada a la intoxicación, y "exposición" al contacto o ingesta de un agente tóxico independientemente de la circunstancia de la misma.

Se analizaron las siguientes variables:

- Demográficas, edad y sexo: se definieron los siguientes grupos etarios (por conveniencia, en base a la clasificación utilizada por el INTOX): preescolares (0-5 años), escolares (6-12 años), adolescentes (13-18 años) y adultos (19-69 años) y adulto mayor ( $\geq 70$ años).

- Circunstancia de la intoxicación: definidas según clasificación utilizada por el CIAT: intencional o suicidio, accidental, delictiva (homicidio o maltrato químico), automedicación y error terapéutico. Se excluyeron las consultas que se catalogan luego del análisis primario como reacción adversa a medicamento o falla terapéutica.

- Severidad del evento: según criterios de la escala Poison Severity Score 23 (PSS) de OMS: leves moderadas y severas $^{(24)}$.

- Evolución de la intoxicación: catalogadas como fatales o no fatales.

- Agente antidepresivo implicado: se incluyeron todos los catalogados como agente antidepresivo según el código Anatomical Therapeutical Classification (ATC) N06A ${ }^{(25)}$ :

- ATC N06AB: inhibidores selectivos de la recaptación de serotonina (ISRS).

- ATC N06AA: antidepresivos tricíclicos (ADT).

- ATC N06AG: inhibidores de la monoaminooxidasa (IMAO).

- ATC N06AX: inhibidores selectivos de la recapación de serotonina y noradrenalina (ISRNS).

- ATC N06AX: otros antidepresivos (mirtazapina, nefazodona, trazodona, bupropión, hipérico, agomelatina).

Se realizó un subanálisis de los eventos severos según sexo, grupo etario, agente antidepresivo implicado y circunstancia de la intoxicación.

Para la descripción de las variables cuantitativas se presentan los datos como media o rango, y para las variables cualitativas se presentan los datos en su frecuen-
Tabla 1. Distribución según sexo y rango etario.

\begin{tabular}{lrc}
\hline Distribución según sexo & & \\
Femenino & 1.904 & $75,4 \%$ \\
Masculino & 619 & $24,6 \%$ \\
Total & 2.523 & $100 \%$ \\
\hline Distribución según edad & & \\
Preescolares (0-5 años) & 112 & $4,4 \%$ \\
Escolares (6-12 años) & 47 & $1,9 \%$ \\
Adolescentes (13-18 años) & 361 & $14,3 \%$ \\
Adultos (19-69 años) & 1.941 & $80 \%$ \\
Adulto mayor ( $\geq 70$ años) & 62 & $2,4 \%$ \\
\hline Total & 2.523 & $100 \%$ \\
\hline
\end{tabular}

cia absoluta y porcentaje para cada nivel de la variable en estudio.

Los datos fueron recogidos de forma digital por un autor integrante del CIAT, anonimizados, y su procesamiento posterior para el análisis de las variables propuestas se realizó en Excel, a cargo de todos los autores.

Este protocolo fue aprobado por el Comité de Ética de la Investigación del Hospital de Clínicas.

\section{Resultados}

Se recibieron 32.565 consultas toxicológicas en el período analizado: los antidepresivos fueron implicados como agente tóxico causal en 2.523 (7,7\%). En 169 de estas consultas $(6,6 \%)$ se vieron implicados más de un medicamento antidepresivo, habiendo diez casos en los que se identificaron tres y en un caso cuatro.

La mayoría de las intoxicaciones ocurrieron en pacientes de sexo femenino $(75,4 \%)$ y adultos $(79,3 \%)$. Se destaca el reporte de casos en menores de 5 años $(4,4 \%)$ (tabla 1).

El antidepresivo más frecuentemente implicado en los eventos de intoxicación fue sertralina (38\%), seguido de venlafaxina $(12,8 \%)$ y fluoxetina $(12,5 \%)$. La distribución completa por tipo de antidepresivo implicado se detalla en la tabla 2.

Con respecto a la circunstancia de las intoxicaciones, un total de 2.279 casos fueron de causa intencional (90\%), de las cuales $2.249(89,1 \%)$ fueron catalogados como suicidio (tabla 3 ).

Del total de las consultas que implicaron un medicamento antidepresivo fueron catalogadas como intoxicaciones severas $54(2,1 \%)$. De ellas, la mayoría corresponde a pacientes de sexo femenino $(n=37,68,5 \%)$ y 


\begin{tabular}{|c|c|c|c|}
\hline Antidepresivo implicado & 2010 & 2011 & 2012 \\
\hline \multicolumn{4}{|l|}{ ATC N06AB: SNRS } \\
\hline Sertralina & 331 & 370 & 327 \\
\hline Fluoxetina & 136 & 121 & 82 \\
\hline Escitalopram & 57 & 71 & 89 \\
\hline Paroxetina & 57 & 62 & 66 \\
\hline Citalopram & 50 & 48 & 39 \\
\hline Fluvoxamina & 26 & 30 & 31 \\
\hline \multicolumn{4}{|l|}{ ATC N06AA: ADT } \\
\hline Amitriptilina & 63 & 80 & 60 \\
\hline Imipramina & 12 & 11 & 10 \\
\hline Clomipramina & 10 & 8 & 1 \\
\hline Maprotilina & 2 & 3 & 1 \\
\hline \multicolumn{4}{|l|}{ ATC N06AX: ISRNS } \\
\hline Venlafaxina & 124 & 117 & 104 \\
\hline Duloxetina & 1 & 8 & 5 \\
\hline \multicolumn{4}{|l|}{ ATC N06AG: IMAO } \\
\hline Meclobemida & 0 & 0 & 1 \\
\hline \multicolumn{4}{|c|}{ ATC N06AX: Otros antidepresivos } \\
\hline Bupropión & 20 & 22 & 19 \\
\hline Trazodona & 6 & 5 & 3 \\
\hline Mirtazapina & 3 & 2 & 1 \\
\hline Hipérico & 1 & 1 & 1 \\
\hline Agomelatina & 0 & 1 & 0 \\
\hline Total & 899 & 960 & 844 \\
\hline
\end{tabular}

fueron intencionales o con fines de autoeliminación $(\mathrm{n}=51,98,1 \%)$. Uno de los casos fue catalogado como maltrato químico, ocurriendo en un escolar de 7 años y en dos se desconoce la causa. Todos los casos severos registrados correspondieron a intoxicaciones mixtas, es decir asociaciones con otros medicamentos o con bebidas alcohólicas (tabla 4). Los antidepresivos más implicados en los casos severos fueron los ISRS (77\%), seguidos de ADT (29\%). Un caso severo evolucionó a la muerte; se trataba de un hombre de 48 años, con depresión de conciencia leve. La causa de la ingesta fue inten-
Tabla 3. Circunstancia de intoxicación.

\begin{tabular}{lc|}
\hline Circunstancia de intoxicación & Frecuencia \\
\hline Intencional & $2.279(90,32 \%)$ \\
Suicidio & $2.249(89,14 \%)$ \\
Abuso & $28(1,10 \%)$ \\
Aborto & $1(0,04 \%)$ \\
Maltrato químico & $1(0,04 \%)$ \\
No intencional & $160(6,34 \%)$ \\
Accidental & $127(5,03 \%)$ \\
Error de medicación & $29(1,15 \%)$ \\
Automedicación & $4(0,16 \%)$ \\
Sin dato & $84(3,34 \%$ \\
\hline Total & $2.523(100 \%)$ \\
\hline
\end{tabular}

to de autoeliminación. Los fármacos implicados en este caso fueron: amitriptilina $(750 \mathrm{mg})$, paracetamol $(15 \mathrm{~g})$, alopurinol $(4,5 \mathrm{~g})$, ácido valproico $(2,5 \mathrm{~g})$ y hierro elemental $(5 \mathrm{mg})$ de un medicamento a base de sulfato ferroso. Falleció en menos de 24 horas como consecuencia de un shock hemodinámico por hemorragia digestiva aguda.

\section{Discusión}

Los antidepresivos fueron catalogados como agentes causales de intoxicaciones en 7,7\% del total de los casos reportados y registrados en la base de datos de intoxicaciones del CIAT a nivel nacional durante el período analizado. Este porcentaje equivale a dos intoxicaciones por antidepresivos por día. Comparativamente se encuentra entre los valores hallados a nivel internacional. En el reporte anual de 2012 de la American Association of Poison Control Centers (AAPCC) se evidenció que $3,75 \%$ del total de las llamadas por intoxicaciones involucró a un medicamento antidepresivo. Esta cifra coincide con la reportada por el Centro Toxicológico de referencia de Rosario, Argentina, que publicó una frecuencia promedio de $3 \%$ de participación de antidepresivos como agentes tóxicos implicados en el período 2000-2009. En España, en un estudio realizado en el centro universitario de toxicología del Hospital Ramón y Cajal de Madrid se registró $16 \%$ de intoxicaciones medicamentosas que implicaban al menos un antidepresivo. Datos obtenidos de un estudio realizado en Santiago de Cuba, donde se describieron las intoxicaciones 


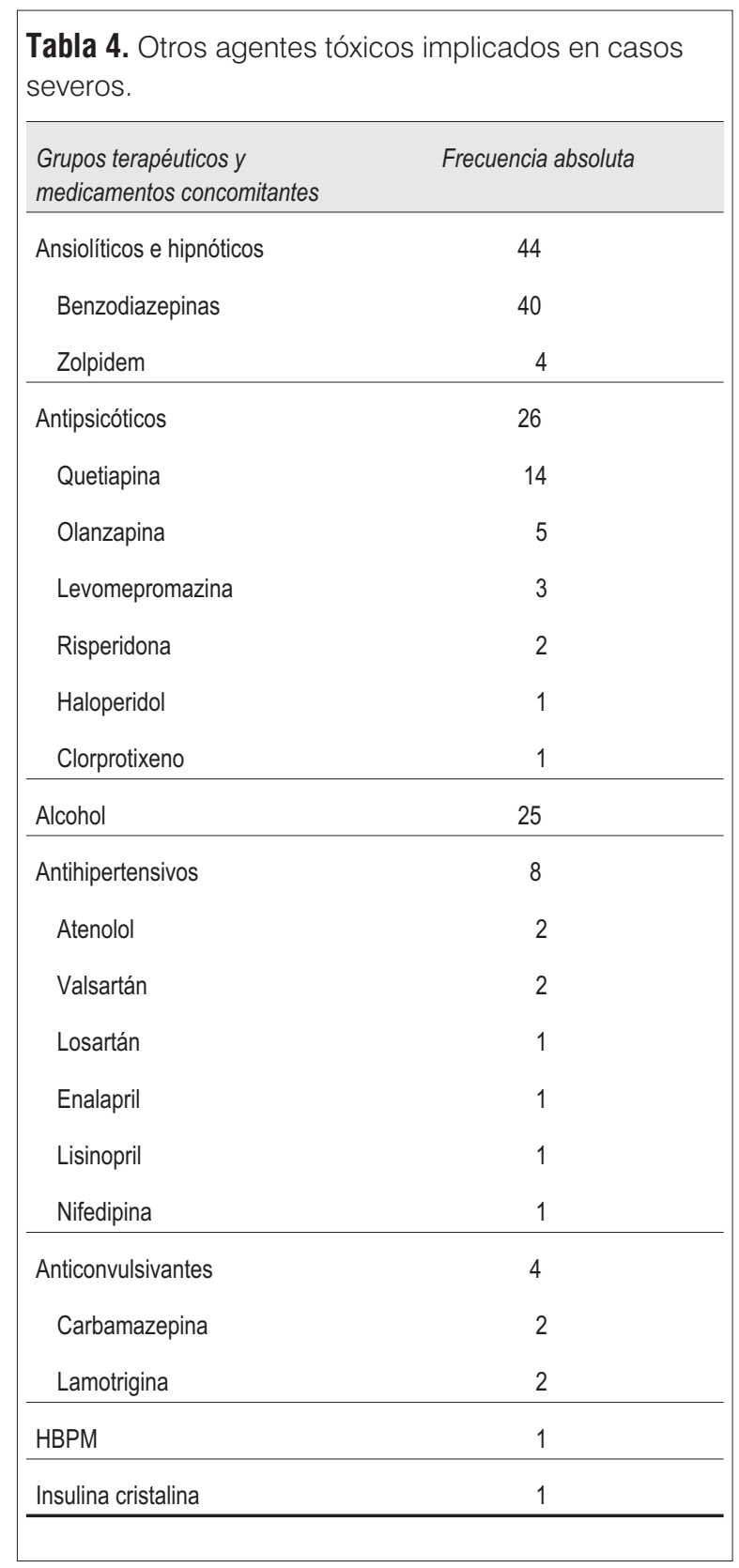

por psicofármacos en un servicio de emergencia, se registró una frecuencia de $17,4 \%$ de antidepresivos implicados $^{(17-20)}$.

En el estudio previo realizado por los autores, las benzodiazepinas fueron catalogadas como agente causal en $28,8 \%$ de los casos, por lo que, comparativamente, los antidepresivos tuvieron en este estudio un papel menos relevante en la generación de intoxicaciones. La percepción de los autores en relación al lugar que ocupaban las benzodiazepinas en las intoxicaciones fue que podría estar determinado por una naturalización de su uso y fácil acceso, independientemente de si era el usuario final o no de dicho medicamento ${ }^{(23)}$.

No obstante, para profundizar el análisis de este aspecto sería necesario conocer el modo en el que accedieron a estos medicamentos los individuos implicados. El estudio recoge la circunstancia de la intoxicación, pero no el cómo llegó ese medicamento a manos del intoxicado (esencialmente si había mediado una indicación médica y por qué motivo). El único dato que aporta la circunstancia de intoxicación es el porcentaje de automedicados. En el estudio de intoxicaciones con benzodiazepinas hubo 35 automedicados en un total de 6.186 casos $(0,56 \%)$ y en este estudio hubo $4 / 2.523(0,16 \%)$, lo que implica un mayor número de automedicados con benzodiazepinas que con antidepresivos $(\mathrm{p}=0,01)$.

La mayoría de los casos de intoxicaciones por antidepresivos que implicaron antidepresivos como agente causal corresponden a personas adultas de sexo femenino, con intención de autoeliminación, lo que coincide con otros reportes a nivel internacional ${ }^{(17-20)}$.

Un dato de suma importancia es que se identificaron 520 casos en menores de 18 años, de los cuales 112 $(21,5 \%)$ fueron en menores de 6 años. De todos los antidepresivos consultados, solo sertralina, fluoxetina y fluvoxamina se encuentran aprobados para su uso en pediatría, según las fichas técnicas disponibles en la AEMPS. En ningún caso se encuentra aprobado el uso de antidepresivos en menores de 6 años. Esto hace suponer dos posibles explicaciones: indicación fuera de prospecto o acceso intra o peridomiciliario de estos medicamentos. El uso fuera de prospecto, posible y algunas veces necesario, implica, no obstante, menor respaldo científico de sus potenciales beneficios y mayor probabilidad de efectos adversos y errores de medicación y menor percepción de estos riesgos ${ }^{(26)}$. La exposición domiciliaria podría ser accidental o intencional, destacándose que en este estudio se identificó un caso intencional, por maltrato químico en un niño de 7 años que además fue catalogado como severo. Con respecto al anterior trabajo de los autores sobre la exposición a benzodiazepinas en niños, en este estudio fue menor, lo que acompaña la hipótesis de la menor utilización de antidepresivos a nivel general mencionada anteriormente.

Los antidepresivos identificados con mayor frecuencia fueron los ISRS, lo que coincide con el perfil de uso actual de antidepresivos y las recomendaciones actuales de tratamiento de la depresión ${ }^{(27)}$. Además, los ISRS han ampliado su uso a otras patologías como trastornos de alimentación, algunas disfunciones sexuales, tratamiento de los sofocos en la menopausia, síndrome premenstrual, correspondiendo algunos de ellos a indi- 
caciones no autorizadas en ficha técnica, pero que hacen al aumento progresivo en el consumo de esos medicamentos que se ve reflejado también en la incidencia de intoxicaciones $^{(28-30)}$.

En cuanto a la severidad, el perfil hallado en el trabajo muestra que son intoxicaciones poco severas y los casos graves estuvieron asociados a la concomitancia de exposición a otras sustancias. Este hecho refleja el perfil de seguridad diferente de los antidepresivos más utilizados, es decir los ISRS (llamados de segunda generación), con respecto a los de primera generación, los cuales se asocian a rangos terapéuticos más estrechos, y efectos adversos cardiovasculares y neurológicos más riesgosos $^{(31)}$.

Entre las limitantes más importantes de la investigación se destaca la imposibilidad, a la fecha de la publicación de este trabajo, de poder evaluar más años en la base de datos INTOX, ni realizar un análisis de la evolución de los casos ingresados al sistema. Este fue el motivo por el cual los casos severos se analizaron en forma manual.

Más allá de las limitaciones mencionadas, este tipo de información es útil para contribuir a la caracterización de uso de este grupo terapéutico en nuestro medio y a complementar la evidencia disponible de los riesgos de uso en la práctica clínica habitual.

\section{Conclusiones}

Este trabajo muestra el perfil de intoxicaciones por antidepresivos en Uruguay entre 2010 y 2012. Como elemento de mayor riesgo se destaca la exposición a estos medicamentos en menores de 18 años y sería necesario profundizar la evaluación de las circunstancias, acceso y uso en este grupo etario. No obstante, la mayoría de los casos fueron no severos y ocurrieron en mujeres adultas con fines de autoeliminación. Los ISRS en combinación con otros agentes tóxicos fueron los más utilizados. Este perfil es similar al reportado en series internacionales y apoya el ya conocido riesgo de los antidepresivos de segunda generación.

\section{Bibliografía}

1. Organización Mundial de la Salud. Notas descriptivas: depresión. Ginebra: OMS, 2012. Disponible en: http://www.who.int/es/news-room/fact-sheets/detail/depression [Consulta: julio 2015].

2. Stork C. Serotonin reuptake inhibitors and atypical antidepressants. En: Hoffman R, Howland M, Lewin N, Nelson L, Goldfrank L. Goldfrank's Toxicologic emergencies. $10^{\text {th }}$ ed. New York: McGraw Hill, 2014:1018-25.

3. Aquines C, Wschebor M, García S. Trastornos del Humor. Trastornos Depresivos. En: Aquines C, comp. Temas de psi- quiatría: manual de psiquiatría para médicos. Montevideo: Oficina del libro Fefmur, 2013.

4. Baldessarini R. Fármacos y tratamiento para trastornos psiquiátricos: depresión y trastornos de ansiedad. En: Goodman \& Gilman. Las bases farmacológicas de la terapéutica. $10^{\mathrm{a}} \mathrm{ed}$. México: Mc Graw Hill, 2001:455-92.

5. Agencia española de medicamentos y productos sanitarios. Ficha técnica: Sertralina. Madrid: AEMPS, 2014. Disponible en: https://www.aemps.gob.es/cima/pdfs/es/ft/681 40/FT_68140.pdf [Consulta: julio 2016].

6. Agencia española de medicamentos y productos sanitarios. Ficha técnica: Paroxetina. Madrid: AEMPS, 2014. Disponible en: https://www.aemps.gob.es/cima/pdfs/es/ft/ 66218/66218_ft.pdf [Consulta: julio 2016].

7. Agencia española de medicamentos y productos sanitarios. Ficha técnica Fluoxetina. Madrid: AEMPS, 2015. Disponible en: https://www.aemps.gob.es/cima/dochtml/ft/ 64787/FichaTecnica_64787.html [Consulta: julio 2015].

8. Agencia española de medicamentos y productos sanitarios. Ficha técnica: Amitriptilina. Madrid: AEMPS, 2015. Disponible https://www.aemps.gob.es/cima/pdfs/es/ft/37 130/FT_37130.pdf [Consulta: julio 2015].

9. Agencia española de medicamentos y productos sanitarios. Ficha técnica: Bupropion. Madrid: AEMPS, 2014. Disponible en: https://www.aemps.gob.es/cima/pdfs/es/ft/ 68616/FT_68616.pdf [Consulta: julio 2015].

10. Agencia española de medicamentos y productos sanitarios. Ficha técnica: Venlafaxina. Madrid: AEMPS, 2014. Disponible en: https://www.aemps.gob.es/cima/pdfs/es/ft/ 70279/FichaTecnica_70279.html.pdf [Consulta: julio 2015].

11. Agencia española de medicamentos y productos sanitarios. Ficha técnica: Duloxetina. Madrid: AEMPS, 2014. Disponible en: https://www.aemps.gob.es/cima/pdfs/es/ft/ 79370/FT_79370.pdf [Consulta: julio 2015].

12. Agencia española de medicamentos y productos sanitarios. Informe de utilización de medicamentos: utilización de medicamentos antidepresivos en España durante el periodo 2000-2013. Madrid: AEMPS, 2015. Disponible en: http://www.aemps.gob.es/medicamentosUsoHumano/observatorio/docs/antidepresivos-2000-2013.pdf [Consulta: julio 2016].

13. Spence R, Roberts A, Ariti C, Bardsley M. Focus On: antidepressant prescribing. Trends in the prescribing of antidepressants in primary care. The Health Foundation, may 2014. Disponible en: http://www.health.org.uk/sites/default/files/QualityWatch_FocusOnAndidepressantPrescribing.pdf [Consulta: julio 2015].

14. Jirón M, Machado M, Ruiz I. Consumo de antidepresivos en Chile entre 1992 y 2004. Rev méd Chile 2008; 136:1147-54. Disponible en: http://www.scielo.cl/pdf/rmc/ v136n9/art09.pdf [Consulta: mayo 2016].

15. Uruguay. Presidencia de la República. Junta Nacional de Drogas. Observatorio Uruguayo de Drogas. VI Encuesta Nacional en Hogares sobre Consumo de Drogas. 2016. Disponible en: https://www.gub.uy/jnd/index.php/comunica- 
cion/publicaciones/vi-encuesta-nacional-en-hogares-sobre-consumo-de-drogas-2016 [Consulta: julio 2016].

16. Uruguay. Presidencia de la República. Junta Nacional de Drogas. Observatorio Uruguayo de Drogas. V Encuesta Nacional en Hogares sobre Consumo de Drogas. 2011. Disponible en: https://www.gub.uy/jnd/index.php/comunicacion/publicaciones/v-encuesta-nacional-en-hogares-sobre-consumo-de-drogas-2011 [Consulta: julio 2015].

17. Torres N, Moscoloni N, Guerrero LM, Piola JC. Análisis de correspondencias múltiples y clasificación de coordenadas factoriales para caracterizar autointoxicaciones intencionales atendidas en SERTOX, Rosario, Argentina entre los años 2000 y 2009. Retel 2013; (41):12-38. Disponible en: http://www.sertox.com.ar/img/item_full/41002c.pdf [Consulta: julio 2015].

18. Medina González L, Fuentes Ferrer M, Suárez Llanos J, Arranz Peña M, Ochoa Mangado E. Epidemiología de las intoxicaciones medicamentosas durante un año en el Hospital Universitario Ramón y Cajal. Rev Clin Esp 2008; 208(9):432-6. Disponible en: http://www.revclinesp.es/es/ pdf/S0014256508760132/S300/ [Consulta: julio 2015].

19. Puente Maury V, Zayas Monteagut J, Mora Linares O, Leyva Suárez N, González Guerrero L. Caracterización de pacientes con intoxicación aguda por psicofármacos. MEDISAN 2013; 17(3):514-20. Disponible en: http://scielo.sld.cu/scielo.php?script=sci_arttext\&pid=S1029-3019201 3000300013 [Consulta: julio 2015].

20. Mowry JB, Spyker DA, Cantilena LR Jr, Bailey JE, Ford M. 2012 Annual Report of the American Association of Poison Control Centers' National Poison Data System (NPDS): 30th Annual Report. Clin Toxicol (Phila) 2013; -51(10):949-1229.

21. Liebelt, E. Cyclic antidepressants. En: Nelson L, Lewin N, Howland M, Hoffman R, Goldfrank L, Flomenbaum N. Goldfrank's Toxicologic emergencies. $9^{\text {th }}$ ed. New York: Mc Graw Hill Education, 2011:1049-57.

22. Stork C. Serotonin reuptake inhibitors and atypical antidepressants. En: Nelson L, Lewin N, Howland M, Hoffman R, Goldfrank L, Flomenbaum N. Goldfrank's Toxicologic emergencies. $9^{\text {th }}$ ed. New York: Mc Graw Hill Education, 2011:1037-45.

23. Domínguez Trobo V, Tortorella MN, Speranza N, Amigo C, Laborde L, Goyret A, Tamosiunas G. Perfil epidemiológico de las intoxicaciones por benzodiazepinas recibidas en el Centro de Información y Asesoramiento Toxicológico uruguayo en el período 2010-2011. Rev Méd Urug 2015; 31(1):32-8.

24. Persson HE, Sjöberg GK, Haines JA, Pronczuk de Garbino J. Poisoning severity score. Grading of acute poisoning. J Toxicol Clin Toxicol 1998; 36(3):205-13.

25. WHO Collaborating Centre for Drug Statistics Methodology. Norwegian Institute of Public Health. ATC/DDD Index. 2017 Disponible en: http://www.whocc.no/atc_ddd index/ [Consulta: mayo 2016].

26. Sperenza N, Tamosiunas G. Uso de medicamentos fuera de prospecto. Uso off label de medicamentos: ¿un mal necesario?. Bol farmacol 2016; 7(2):6p. Disponible en: http://www.boletinfarmacologia.hc.edu.uy/images/stories/boletin/uso_de_medicamentos_fuera_de_prospecto.pdf [Consulta: marzo 2017].

27. Ministerio de Sanidad, Servicios Sociales e Igualdad. Agencia de Evaluación de Tecnologías Sanitarias de Galicia. Guía de práctica clínica sobre el manejo de la depresión en el adulto. España, 2014. Disponible: http://www.guiasalud.es/GPC/GPC_534_Depresion_Adulto_Avaliat_compl.pdf [Consulta: octubre 2016].

28. Viroga S. ISRS en la menopausia. Tratamiento de los sofocos. Bol farmacol 2016; 7(1): 3 p. Disponible en: http://www.boletinfarmacologia.hc.edu.uy/images/stories/boletin/isrs.pdf [Consulta: marzo 2017].

29. Wyatt KM, Dimmock PW, O'Brien PMS. Inhibidores selectivos de la recaptación de serotonina para el síndrome premenstrual (Revisión Cochrane traducida). Cochrane Plus 2013; (6). Disponible en: https://www.cochrane.org/es/CD001396/inhibidores-selectivos-de-la-recaptacion-de-serotonina-para-el-sindrome-premenstrual [Consulta: marzo 2015].

30. Wespes E, Amar E, Eardley I, Giuliano F, Hatzichristou D, Hatzimouratidis K, et al. Guía clínica sobre la disfunción sexual masculina: disfunción eréctil y eyaculación precoz. European Association of Urology, 2010. Disponible en: https://uroweb.org/wp-content/uploads/12-GUIA-CLINICA-DISFUN-SEXUAL-MAS CULINA.pdf [Consulta: marzo 2016].

31. Pérez V, Speranza N, Tamosiunas G, Ormaechea G. Perfil de riesgo de los antidepresivos inhibidores selectivos de la recaptación de serotonina (ISRS). Rev urug med interna 2016; 3:25-33. 